großen Differenzen, welche die Seeligersche Theorie in 1878 übrig läßt, könnte also eine Folge der Unzulänglichkeit der Theorie sein.

Ihr systematischer Charakter tritt noch schärfer hervor, wenn man sie nach der Phase ordnet und zu Mitteln zusammenzieht :

$\begin{array}{ccc}\alpha & \text { Mittl. Abw. } & \text { Anzahl } \\ 0.48 & -0.05 & (6) \\ 1.75 & 0.00 & (6) \\ 3.90 & +0.10 & (6) \\ 4.68 & +0.07 & (6) \\ 5.43 & +0.13 & (7)\end{array}$

Eine Abhängigkeit von $\alpha$, mit einem Koeffizienten $0.035 \pm 0.006$ ist in diesen Zahlen deutlich enthalten.

In der Opposition I $879 / 80$ findet Müller einen kleineren Koeffizienten als in den anderen Jahren, obgleich noch größer als die Theorie erheischt; die Differenz ist jedoch schwerlich zu verbürgen und die Abweichungen $B-R$ zeigen nichts systematisches.

Wenn der Phasenkoeffizient nahezu konstant ist, unabhängig von $A$, wie Müller es in seiner empirischen Darstellung annahm, so bedeutet das, daß nicht nur der Ring, sondern auch das Saturnssphäroid einen bedeutenden Phaseneinfluß erleidet, von derselben Größenordnung wie den des Ringes. Nun sind die Helligkeitsdifferenzen, auf denen dieses Resultat ruht, sehr gering im Verhältnis zu der Genauigkeit photometrischer Messungen; man wird es also gar nicht als fest verbürgt ansehen können. Es gibt aber noch einige andere Umstände, die auf dasselbe hinweisen, obgleich sie ebensowenig die Realität des Phänomens beweisen können:

I) Die wenigen Messungen in 1877 , bei sehr kleinen $A$ und $A^{\prime}$ geben bei großem Phasenwinkel alle. eine geringere Helligkeit als die Theorie fordert, während die Müllersche empirische Darstellung kleinere Abweichungen von wechselndem Zeichen ưbrig lältt.

Leiden, 1905 Januar.
2) Auch die Beobachtungen aus 189 I bei kleinen $A$ und $A^{\prime}$ werden von den Müllerschen Tafeln besser dargestellt als von der Theorie; obgleich die Phase nur $2: 63$ erreicht, ist doch ein Phaseneinfluß angedeutet.

3) Die von Zöllner im Jahre 1862 bei verschwundenem Ringe angestellten Messungen sind, wie Müller S. 343 nachweist, nur durch eine bedeutende Phasenkorrektion mit der Müllerschen Normalgröße und mit seinen eigenen Resultaten von $1863-65$ in Einklang zu bringen.

Dies alles würde darauf hinweisen, daß die von Seeliger gegebene Theorie eine Korrektion in dem Sinne zu erhalten hat, daß der Saturnskörper selbst einen Phaseneinfluß von einigen Hundertstel Größen pro Grad erleidet, so daß dem Ringe ein kleinerer Beitrag zu dem Phasenkoeffizient obliegt als bisher. Damit geht wohl ein Vorzug der Theorie verloren, nämlich der, daß sie, nur von physischen Verhältnissen ausgehend, die Lichtänderungen des Saturn rein theoretisch voraussagen kann; es kommt ein empirisches Glied hinein, das man theoretisch nicht erklären kann. Zwar ist dies kein Grund, von vornherein den hier angedeuteten Sachverhalt unmöglich zu erachten, denn es kommen gleich große Koeffizienten bei den kleinen Planeten vor (Ceres 0.042 , Merkur 0.037 pro Grad); dennoch erscheint es seltsam, dalb Saturn in bezug auf seine Oberflächenbeschaffenheit nicht bei Jupiter, sondern bei Merkur, dem Monde und den kleinen Planeten eingereiht werden soll.

Darum ist eine Entscheidung durch neue photometrische Messungen von großer Wichtigkeit. Die hier zusammengestellten Andeutungen sind zu schwach, um etwas beweisen zu können, und bei der Geringfügigkeit der Differenzen, um die es sich handelt, wäre es unzulässig, schon jetzt die Unzulänglichkeit der Theorie behaupten zu wollen. Die nächsten Jahre werden aber Gelegenheit bieten, eine Entscheidung zu treffen; im Jahre 1907 wird der Ring verschwinden, und in den Oppositionen von 1905 und 1906 wird die Elevation ungefähr der der Oppositionen von 1879 und 1878 gleich sein.

Ant. Pannekoek.

\title{
Zusatz von Herrn Professor H. Seeliger.
}

Schon Herr G. Muiller hat darauf hingewiesen, daß die photometrische Beobachtung des ringlosen Saturn im Jahre 1907 die Lücke, die in unserer Kenntnis von den Lichtverhältnissen des Saturnsystems gegenwärtig noch besteht \& ausfullen kann, und wenn Herr Pannekoek von neuem darauf aufmerksam macht, so ist dies gewiß sehr dankenswert.

Ich möchte aber bei dieser Gelegenheit daran erinnern, daß die Theorie der Beleuchtung des Saturn ringes, da sie mit sehr komplexen Verhältnissen, die nur angenähert berücksichtigt werden können, zu rechnen hat, nur beanspruchen kann, die tatsächlichen Verhältnisse in großen Zügen darzustellen, auch wenn die eingehenden Konstanten besser, als bisher durchfuibrbar war, bestimmt sein werden. Jedenfalls müßte bei einer etwaigen detaillierteren Vergleichung auf die Verschiedenheit der einzelnen Ringteile in optischer Beziehung geachtet werden und bei kleineren Elevationswinkeln kann, wie ich in meiner zweiten Abhandlung (München 1893) erörtert habe, die Beleuchtung des Florringes Einfluß ge-

winnen. Dazu kommt noch ein Umstand, den man in Rücksicht $z$ u ziehen haben wird.

Die verdienstvollen Beobachtungen des Herrn G. Müller haben bekanntlich gezeigt, daß die Helligkeit einiger der kleinen Planeten ganz abnorm starke Einwirkungen der Phase, und zwar auch bei kleinen Phasenwinkeln, zeigen. Man muß, wie ich bei andern Gelegenheiten auseinandergesetzt habe, annehmen, daß ähnliches überhaupt bei rauhen von der Sonne beleuchteten kosmischen Körpern stattfinden wird. Daraus folgt, daß der Phasenfaktor $f(\alpha)$ in der Theorie der Beleuchtung des Saturnringes wahrscheinlich nicht konstant zu lassen ist, welche Kompletierung natürlich ohne Mühe auszuführen ist, wenngleich die Einführung eines solchen mehr oder weniger empirischen Gliedes immerhin mit Unsicherheiten behaftel sein wird. Daß der Saturnring aus solchen Teilchen zusammengesetzt ist, welche zu der Kategorie der eben erwähnten gehören, ist wohl kaum zu bezweifeln. Welchen Betrag dieser Faktor hat, kann nur durch eine sehr eingehende Diskussion ermittelt werden.

Sternwarte München, I $9 \circ 5$ Febr. 9. 